\title{
Modelamiento Matemático y Simulación de un Reactor Rotatorio Industrial para la Producción de Clinker
}

\author{
Jesús D. Coral(1), Izabela Dobrosz-Gómez ${ }^{(2)}$ y Miguel Á. Gómez ${ }^{(1)}$ \\ Universidad Nacional de Colombia, Sede Manizales, (1) Departamento de Ingeniería Química, \\ (2) Departamento de Física y Química, PRISMA: Campus La Nubia, km 9 vía al Aeropuerto la Nubia, \\ A. A. 127, Manizales, Caldas, Colombia (e-mail: jdcoralm@unal.edu.co, idobrosz-gomez@unal.edu.co, \\ magomez@unal.edu.co)
}

Recibido Dic. 16, 2013; Aceptado Mar. 17, 2014; Versión final recibida Mar. 27, 2014

\begin{abstract}
Resumen
En este trabajo se propone un modelo matemático para simular un reactor rotatorio industrial para la producción de cemento en bruto (clinker). El modelo unidimensional incluye la variación del movimiento de los sólidos a lo largo del reactor, los balances de conservación de materia y energía para las fases sólida y gaseosa y un modelo para estimar el consumo de combustible. Se aplicó el método del disparo para la solución iterativa del modelo propuesto. Se determinaron los perfiles de concentración de reactivos y productos, y de temperatura, en la fase sólida y gaseosa, con desviaciones inferiores al $7 \%$. Adicionalmente, fue posible cuantificar el fenómeno de inversión térmica que ocurre cuando el material fundido se enfría después del máximo de temperatura. Basado en los resultados, se concluye que el modelo propuesto constituye una herramienta útil para el análisis del desempeño del reactor rotatorio.
\end{abstract}

\section{Mathematical Modeling and Simulation of an Industrial Rotary Reactor for Clinker Production}

\begin{abstract}
This paper summarizes the results on the modeling and simulation of an industrial rotatory kiln for clinker production. The proposed one-dimensional model includes several sub-models for simulating the variation of the bed height in the kiln, the reactive mass and energy balances in the solid and gas phases and the coal combustion. The shooting method was chosen for iteratively solving the coupled models. The model predictions of both reactant and products concentrations and gas and solid phase temperatures are in good agreement with the industrial data within deviations lower than $7 \%$. Additionally, it was possible to quantify the thermal inversion phenomena, which occurs when the melt gets colder after having passed the peak temperature. Based on the results, it can be concluded that the proposed model is a useful tool for analyzing the performance of the rotatory kiln.
\end{abstract}

Keywords: rotatory kiln, clinker production, one-dimensional model, shooting method 


\section{INTRODUCCIÓN}

El clinker - componente base del cemento - es un material clave en el mundo industrial. Su tecnología de fabricación, basada en el uso de reactores rotatorios, se ha visto obligada constantemente a modernizarse con el fin de cumplir simultáneamente las cada vez más severas legislaciones ambientales (ya que por tonelada de clinker se emiten entre $900-1000 \mathrm{~kg}$ de $\mathrm{CO}_{2}$ y 0.3-4.7 $\mathrm{kg} \mathrm{NO}$ ), a aumentar su capacidad de producción (hasta 10000 ton/día en algunas plantas en Asia) y a reducir los consumos energéticos ( 3000$4000 \mathrm{MJ} /$ ton de clinker) para mejorar su rentabilidad (European Commission, 2013). Así, durante las últimas décadas, esta tecnología ha cambiado de procesos de producción húmedos a secos, se han diferenciado las zonas de reacción (calcinación luego clinkerización), se han diseñado sistemas de recuperación de energía y las unidades de control de emisiones son de última tecnología. Sin embargo, la producción de cemento continúa siendo un sector altamente contaminante y energíboro.

Los reactores rotatorios son unidades de proceso de gran tamaño (longitudes de 40 a $70 \mathrm{~m}$ y diámetros que varían entre 1.5 y $3 \mathrm{~m}$ ). Estos equipos se encuentran inclinados (entre $1^{\circ}$ y $3^{\circ}$ ) y girando sobre su propio eje (de $2-6 \mathrm{rpm}$ ) con el fin de facilitar el transporte del material sólido. Adicionalmente, están revestidos de diversas capas de material aislante térmico y tienen instalado un quemador en la sección final del equipo. El material sólido rocoso que ingresa al proceso está conformado principalmente por una mezcla de carbonato de calcio $\left(\mathrm{CaCO}_{3}\right)$, óxido de silicio $\left(\mathrm{SiO}_{2}\right)$, óxido de aluminio $\left(\mathrm{Al}_{2} \mathrm{O}_{3}\right)$ y óxido de hierro $\left(\mathrm{Fe}_{2} \mathrm{O}_{3}\right)$. Antes de ingresar al reactor rotatorio, este material es parcialmente calcinado. Entre 40 y $60 \%$ de la calcinación se logra en los pre-calentadores, para luego desarrollar un proceso químico de pirólisis (a temperaturas entre los 900$1500^{\circ} \mathrm{C}$ ) que da origen al clinker (Kunii y Chisaki, 2008).

La simulación de estos equipos es de especial interés debido a que sus condiciones de operación dificultan el desarrollo de estudios experimentales. Diferentes enfoques se encuentran disponibles en la literatura. La dinámica de fluidos computacional (CFD por sus siglas en inglés) es una de las herramientas más recientes (Mujumdar y Ranade, 2008). Ella permite obtener una visión bidimensional del funcionamiento del reactor rotatorio, e inclusive del funcionamiento del quemador (Fidaros et al., 2007). Sin embargo, la CFD tiene importantes limitaciones tales como el limitado acople de las condiciones de frontera entre el reactor y las fases reactivas (lo que afecta los resultados de temperatura de la fase gas y el flujo de calor) y el excesivo tiempo de cómputo (por ejemplo, Mastorakos et al. (1999) consideraron 47 y 155 nodos en la dirección radial y axial, respectivamente, reportando tiempos de cómputo entre 10 horas y 10 días). Los modelos bidimensionales, basados en el método numérico de los elementos finitos, también permiten realizar el análisis no lineal de un reactor rotario, y aunque los tiempo de cómputo no son tan prolongados, conservan parte de las limitaciones de la CFD (Coz Díaz et al., 2002). Finalmente, los modelos unidimensionales permiten un balance apropiado entre la velocidad de cálculo (especialmente útil para el control de procesos) y la precisión del modelamiento. Su uso está teóricamente fundamentado en la baja relación diámetro-longitud (<10) y los números de Peclet mayores a $10^{4}$ que presentan estos equipos (Froment et al., 2011).

Por lo tanto, en este trabajo se propone un modelo unidimensional eficiente para simular el comportamiento de reactores rotatorios industriales. El modelo tiene en cuenta el movimiento del material sólido, la variación del flujo de los sólidos, el proceso reactivo en la fase sólida, la transferencia de masa y energía entre los gases, el lecho de sólidos y las paredes internas del reactor, y un modelo de encogimiento de partícula para simular el consumo del combustible sólido alimentado a los quemadores del horno. Los resultados obtenidos, mediante simulación por computadora, se validaron con mediciones de planta suministradas por una empresa cementera colombiana. Estos permiten obtener un mejor entendimiento de la influencia de las condiciones de operación sobre el desempeño del reactor rotatorio.

\section{MODELO MATEMÁTICO}

El modelo propuesto incluye la variación de la altura del material sólido en el interior del reactor rotatorio, balances de materia y energía en las fases sólida y gaseosa y un modelo cinético gas-sólido para el combustible. Se consideró que la transferencia de materia entre el lecho de sólidos y los gases de combustión es ocasionada principalmente por la migración de $\mathrm{CO}_{2}$ (producto de la calcinación de carbonado de calcio). En la Fig. 1 se presentan las características incluidas en el modelo unidimensional propuesto en este estudio.

Los modelos individuales fueron acoplados entre sí a través de la comunicación fronteras comunes de transferencia de masa y energía. Estos se detallan a continuación. 


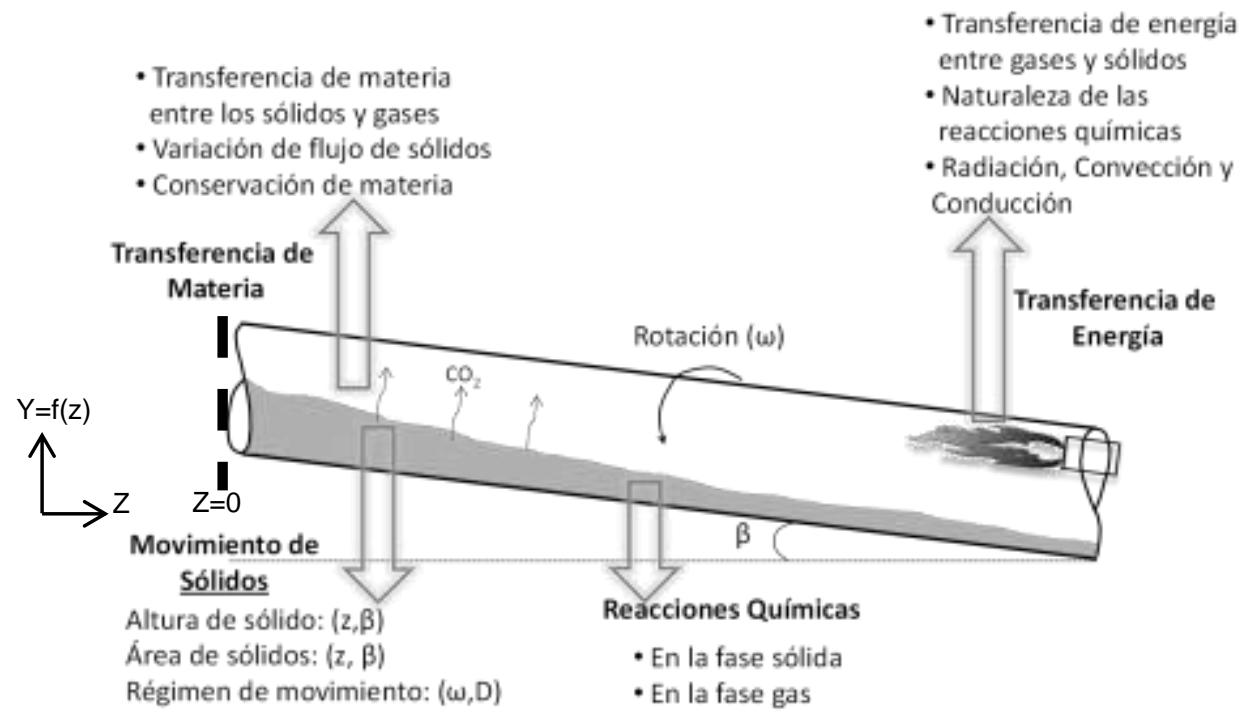

Fig. 1. Elementos incluidos en la simulación unidimensional del reactor rotario industrial para la producción de clinker

\section{MODELO PARA LA FASE SÓLIDA}

El modelo que describe el comportamiento de la fase sólida tiene en cuenta la variación de la altura del lecho de sólidos, ecuaciones de conservación de materia y energía y reducción de flujo.

\section{PERFIL DE ALTURA Y ÁREA OCUPADA DEL LECHO DE SÓLIDOS}

La estimación precisa del área ocupada por el lecho de sólidos se llevó a cabo a partir del modelo propuesto de Saeman (Kunii y Chisaki, 2008). Este se presenta en la ecuación (1).

$$
d A_{s}=2 r \sin ^{-1}\left(\frac{\left(r^{2}-r_{0}^{2}\right)^{1 / 2}}{r}\right) d r
$$

La variación del radio mínimo de giro ( $r_{0}$ ) con la posición axial ( $\mathrm{z}$ ) se calcula mediante la ecuación (2).

$$
\frac{d r_{0}}{d z}=\frac{\left(3 q_{s} \sin (\Theta)\right)}{4 \pi \omega\left(R_{H}^{2}-r_{0}^{2}\right)^{3 / 2} \cos (\Theta)}-\frac{\beta}{\cos (\Theta)}
$$

Por lo tanto, a partir de las ecuaciones (2) y (3) se puede conocer la variación de la profundidad del lecho de sólidos.

$$
h_{s}=R_{H}-r_{0}
$$

\section{BALANCES DE MATERIA Y ENERGÍA PARA EL LECHO DE SÓLIDOS}

El balance de materia para cada especie en la fase sólida se puede escribir de la siguiente manera:

$$
\frac{d\left(A_{s} \rho_{s} u_{s} Y_{i s}\right)}{d z}=R_{\text {is }} A_{s}
$$

Para determinar la reducción del flujo de material sólido, se supuso que el único compuesto gaseoso producido en la calcinación es el $\mathrm{CO}_{2}$, como se muestra en la ecuación (5): 


$$
\mathrm{F}_{\mathrm{s}}^{\mathrm{z}}=\mathrm{F}_{\mathrm{s}}^{\mathrm{z}-\Delta z}-\mathrm{Y}_{\mathrm{CO}_{2}}^{\mathrm{z}} \mathrm{F}_{\mathrm{s}}^{\mathrm{z}-\Delta z}
$$

El balance de energía tiene en cuenta la variación de la densidad del lecho de sólidos y la migración de $\mathrm{CO}_{2}$ hacia la fase gas (Martins et al., 2001). Este se presenta en la ecuación (6).

$$
\frac{d T_{s}}{d z}=\frac{Q_{s}+\sum_{i=1}^{N R} \Delta H_{r x n_{i s}}\left(T_{s}\right) R_{i s}-Q_{C O_{2}}}{A_{s} \rho_{s} u_{s} C p_{s}}-\left[T_{s}+\frac{\lambda_{m l}}{C p_{s}}\right]\left[\frac{1}{\rho_{s}} \frac{d \rho_{s}}{d z}+\frac{1}{A_{s}} \frac{d A_{s}}{d z}\right]
$$

\section{MODELO PARA LA FASE GAS}

El balance de materia para cada componente en fase gaseosa queda de la siguiente forma:

$$
\begin{aligned}
& \frac{d Y_{i G}}{d z}=\frac{R_{j G}-Q_{\mathrm{CO}_{2}}}{A_{G} u_{G} \rho_{G}}+F_{\mathrm{CO}_{2}} \\
& F_{\mathrm{CO}_{2}}=\frac{R_{\mathrm{CO}_{2} \mathrm{~S}}}{u_{G} \rho_{G}}
\end{aligned}
$$

El balance de energía, que tiene en cuenta la energía que llega con el flujo de $\mathrm{CO}_{2}$ desde el lecho de sólidos y la transferencia de energía entre los gases calientes, las paredes del reactor y el lecho de material sólido, corresponde a la ecuación (9).

$$
\frac{d T_{G}}{d z}=\frac{1}{\sum F_{i G} C p_{i G}}\left[F_{\mathrm{CO}_{2}} H_{\mathrm{CO}_{2}}\left(T_{s}\right)-Q+A_{G} \sum_{i=1}^{N R} \Delta H_{r \times n_{i G}}\left(T_{G}\right) R_{i G}\right]
$$

\section{REACCIONES EN FASE SÓLIDA}

El proceso reactivo de formación de clinker es bastante complejo. Se han reportado más de 30 reacciones que ocurren en el lecho de sólidos (Taylor, 1997; Hewlett, 1988). Estas son homogéneas (sólido-sólido y líquido-líquido) y heterogéneas (sólido-líquido). Sin embargo, de acuerdo con la composición de clinker suministrado por empresa bajo estudio y que corresponde con la norma ASTM 150 (astm.org, 2013), el proceso reactivo se podría modelar considerando solo las 5 principales reacciones químicas en la fase sólida. En la Tabla 1 se presentan las reacciones consideradas en este trabajo y sus calores estándar de reacción.

Tabla 1. Reacciones, calores de reacción estándar y parámetros cinéticos

\begin{tabular}{llccc}
\hline \multicolumn{1}{c}{ Reacción } & $\Delta \mathrm{H}_{\mathrm{rnn}, \mathrm{s}}, \mathrm{kJ} / \mathrm{mol}$ & $\mathrm{K}$ & $\mathrm{E}, \mathrm{kJ} / \mathrm{mol}$ \\
\hline 1 & $\mathrm{CaCO}_{3} \rightarrow \mathrm{CaO}^{2} \mathrm{CO}_{2}$ & 179.4 & $1.18 \times 10^{3} \mathrm{kmol} /\left(\mathrm{m}^{2} . \mathrm{s}\right)$ & 179.4 \\
2 & $2 \mathrm{CaO}_{2} \mathrm{SiO}_{2} \rightarrow \mathrm{Ca}_{2} \mathrm{SiO}_{4}\left(\mathrm{C}_{2} \mathrm{~S}=\right.$ Belita $)$ & -127.6 & $1 \times 10^{7} \mathrm{~m}^{3} /(\mathrm{kg} . \mathrm{s})$ & -127.6 \\
3 & $\mathrm{Ca}_{2} \mathrm{SiO}_{4}+\mathrm{CaO}_{2} \rightarrow \mathrm{Ca}_{3} \mathrm{SiO}_{5}\left(\mathrm{C}_{3} \mathrm{~S}=\right.$ Alita $)$ & 16 & $1 \times 10^{9} \mathrm{~m}^{3} /(\mathrm{kg} . \mathrm{s})$ & 16.0 \\
4 & $3 \mathrm{CaO}+\mathrm{Al}_{2} \mathrm{O}_{3} \rightarrow \mathrm{Ca}_{3} \mathrm{Al}_{2} \mathrm{O}_{6}\left(\mathrm{C}_{3} \mathrm{~A}\right)$ & 21.8 & $1 \times 10^{8} \mathrm{~m}^{3} /(\mathrm{kg} . \mathrm{s})$ & 21.8 \\
5 & $4 \mathrm{CaO}+\mathrm{Al}_{2} \mathrm{O}_{3}+\mathrm{Fe}_{2} \mathrm{O}_{3} \rightarrow \mathrm{Ca}_{4} \mathrm{Al}_{2} \mathrm{Fe}_{2} \mathrm{O}_{10}\left(\mathrm{C}_{4} \mathrm{AF}\right)$ & -41.3 & $1 \times 10^{8} \mathrm{~m}^{3} /(\mathrm{kg} . \mathrm{s})$ & -42.3 \\
\hline
\end{tabular}

La velocidad de reacción para estas reacciones se calculó mediante la ecuación (10).

$$
R_{i S}=\sum_{j=1}^{N R} Z_{i j} K_{o j} \exp \left(\frac{E_{j}}{R_{g} T_{S}}\right) \prod_{k=1}^{N C} C_{k}
$$

La ecuación (10) asume que todas las reacciones son de primer orden con respecto a la concentración de cada reactivo y no tiene en cuenta el cambio de fase en las reacciones. 


\section{MODELO CINÉTICO}

El caso industrial bajo estudio utiliza carbón pulverizado como combustible (diámetro de partícula, $\mathrm{d}_{\mathrm{p}}=5.0 \mathrm{x}$ $10^{-5} \mathrm{~m}$ ). Se considera aquí que el material volátil, contenido en el carbón, está compuesto por metano el cual es liberado justo al ingresar a la boquilla del horno (Schaffel et al., 2009). Su oxidación sigue la estequiometria presentada en la Tabla 2 (reacción 1) y la velocidad de reacción según la ecuación (11) (Mujumdar et al., 2006).

$$
\mathrm{R}_{\mathrm{CH}_{4}}=1.6 \times 10^{10} \rho_{\mathrm{G}}^{2} \mathrm{Y}_{\mathrm{C}} \mathrm{Y}_{\mathrm{O}_{2}} \exp \left(-\frac{3.125 \times 10^{6} \mathrm{~J} /(\mathrm{mol} . \mathrm{K})}{\mathrm{R}_{\mathrm{g}} \mathrm{T}_{\mathrm{G}}}\right)
$$

Tabla 2. Reacciones consideradas para la fase gaseosa

\begin{tabular}{clc}
\hline & \multicolumn{1}{c}{ Reacción } & $\Delta \mathrm{H}_{\mathrm{rxn}, \mathrm{G}, \mathrm{kJ} / \mathrm{mol}}$ \\
\hline 1 & $\mathrm{CH}_{4}+2 \mathrm{O}_{2} \rightarrow \mathrm{CO}_{2}+2 \mathrm{H}_{2} \mathrm{O}$ & -802.9 \\
2 & $\mathrm{C}+\mathrm{O}_{2} \rightarrow \mathrm{CO}_{2}$ & -393.8 \\
\hline
\end{tabular}

Para la oxidación de carbón, se consideró un modelo de "encogimiento de coraza" tal como lo propone Levenspiel (1999). Para este proceso, la ley de velocidad de reacción se calculó utilizando las ecuaciones (12) a (15) (Ishii, 2000).

$$
\begin{aligned}
& \frac{1}{\mathrm{R}_{\mathrm{Ox}, \mathrm{C}}}=\frac{1}{\mathrm{~K}_{\mathrm{C}_{1} \mathrm{P}_{\mathrm{O}_{2}}}}+\frac{1}{\mathrm{~K}_{\mathrm{C} 2} \mathrm{P}_{\mathrm{O}_{2}}}+\frac{1}{\mathrm{~K}_{\mathrm{C} 3}} \\
& \mathrm{~K}_{\mathrm{C} 1}=\left[\frac{\rho_{\mathrm{O}_{2}} \mathrm{M}_{\mathrm{c}} \underline{\mathrm{D}}}{\mathrm{M}_{\mathrm{O}_{2}}\left(\mathrm{~d}_{\mathrm{p}} / 2\right)}\right] \mathrm{Nu}\left[\frac{\mathrm{T}_{\mathrm{G}}}{\mathrm{T}_{0}}\right]^{1.5} \\
& \mathrm{~K}_{\mathrm{C} 2}=\left[\frac{\rho_{\mathrm{G}} \mathrm{M}_{\mathrm{c}}}{2 \mathrm{M}_{\mathrm{G}}^{2}}\right]\left[\frac{\mathrm{T}_{0}}{\mathrm{~T}_{\mathrm{G}}}\right]^{0.5} \exp \left(-\frac{14235.12 \mathrm{~J} /(\mathrm{mol} . \mathrm{K})}{\mathrm{R}_{\mathrm{g}} \mathrm{T}_{\mathrm{G}}}\right) \\
& \mathrm{K}_{\mathrm{C} 3}=1.05 \times 10^{5} \exp \left(-\frac{167472 \mathrm{~J} /(\mathrm{mol} . \mathrm{K})}{\mathrm{R}_{\mathrm{g}} \mathrm{T}_{\mathrm{G}}}\right)
\end{aligned}
$$

\section{ECUACIONES DE TRANSFERENCIA DE ENERGÍA}

Dentro de un reactor rotatorio, la transferencia de energía calórica se da a través de los mecanismos de conducción, convección y radiación, predominando esta última. En este trabajo se utilizó el modelo propuesto por Li et al. (2005). Así, el flujo de calor por convección entre los gases calientes y el lecho de sólidos (QcGs) y entre los gases y las paredes internas del reactor (QcGP) se evaluaron usando las ecuaciones (16) a (21).

$$
\begin{aligned}
& \mathrm{Q}_{\mathrm{CGk}}=\mathrm{h}_{\mathrm{CGk}} \mathrm{A}_{\mathrm{CGk}}\left(T_{\mathrm{G}}-\mathrm{T}_{\mathrm{k}}\right), \quad \mathrm{k}=\mathrm{S}, \mathrm{P} \\
& \mathrm{h}_{\mathrm{CGS}}=0.46 \frac{\mathrm{k}_{\mathrm{G}}}{\mathrm{D}_{\mathrm{e}}} \operatorname{Re}_{\mathrm{D}}^{0.535} \mathrm{Re}_{\mathrm{w}}^{0.104}\left(\frac{\beta-\sin (\beta)}{2 \pi}\right)^{-0.341} \\
& \mathrm{~h}_{\mathrm{CGP}}=1.54 \frac{\mathrm{k}_{\mathrm{G}}}{\mathrm{D}_{\mathrm{e}}} \operatorname{Re}_{\mathrm{D}}^{0.575} \mathrm{Re}_{\mathrm{w}}^{-0.292}
\end{aligned}
$$




$$
\begin{aligned}
& D_{e}=\frac{0.5 D(2 \pi-\Theta+\sin (\Theta))}{\left(\pi-\frac{\Theta}{2}+\sin \left(\frac{\Theta}{2}\right)\right)} \\
& \operatorname{Re}_{D}=\frac{D_{e} u_{G} \rho_{G}}{\mu_{G}} \\
& \operatorname{Re}_{w}=\frac{D_{e}^{2} \omega \rho_{G}}{\mu_{G}}
\end{aligned}
$$

El flujo de calor por radiación entre el gas caliente y el material sólido (QRGS), entre el gas caliente y las paredes internas del horno ( $Q_{\mathrm{RGP}}$ ) y entre el lecho de sólidos y las paredes internas del reactor se determinó de las ecuaciones (22) y (23).

$$
\begin{aligned}
& Q_{R G k}=\sigma A_{R G k}\left(\varepsilon_{k}+1\right)\left(\frac{\varepsilon_{G} T_{G}^{4}-\alpha_{G} T_{k}^{4}}{2}\right), \quad k=S, P \\
& Q_{R S P}=\sigma A_{R S P} \varepsilon_{S} \varepsilon_{P}\left(1-\varepsilon_{G}\right)\left(T_{P}^{4}-T_{S}^{4}\right) \frac{L_{S}}{2(\pi-\beta) R}
\end{aligned}
$$

\section{CASO DE ESTUDIO}

El modelo desarrollado fue probado simulando un reactor rotatorio de una empresa cementera colombiana. Las dimensiones del equipo se presentan en la Tabla 3. Los datos de planta seleccionados para desarrollar la simulación y verificar los resultados se presentan en la Tabla 4.

Tabla 3. Dimensiones y parámetros de operación del reactor rotatorio industrial a simular

\begin{tabular}{lc}
\hline Variable & Valor \\
\hline Longitud [m] & 50 \\
Radio interno del reactor [m] & 1,7 \\
Espesor material aislante [m] & 0,068 \\
Espesor segundo material aislante [m] & 0,4 \\
Radio interno coraza [m] & 2,168 \\
Radio externo coraza [m] & 2,2 \\
Velocidad de giro del reactor [r.p.m] & 5,5 \\
Angulo de inclinación [grados] & 3,5 \\
\hline
\end{tabular}

Tabla 4. Datos de planta de los flujos y composiciones de entrada al reactor rotatorio

\begin{tabular}{lclc}
\hline \multicolumn{1}{c}{ Sólidos } & \multicolumn{2}{c}{ Gases } \\
\hline Flujo Total $[\mathrm{Kg} / \mathrm{s}]$ & 33.48 & Flujo Total $[\mathrm{Kg} / \mathrm{s}]$ & 67.65 \\
\hline Composiciones másicas en $\mathrm{z}=\mathrm{L}$ & & Composiciones másicas en $\mathrm{z}=0$ & \\
\hline $\mathrm{CaCO}_{3}$ & 0.36 & $\mathrm{CH}_{4}$ & 0.011 \\
$\mathrm{CaO}$ & 0.39 & $\mathrm{O}_{2}$ & 0.22 \\
$\mathrm{SiO}_{2}$ & 0.21 & $\mathrm{~N}_{2}$ & 0.74 \\
$\mathrm{Al}_{2} \mathrm{O}_{3}$ & 0.0425 & $\mathrm{C}$ (sólido pulverizado) & 0.015 \\
$\mathrm{Fe}_{2} \mathrm{O}_{3}$ & 0.02 & $\mathrm{H}_{2} \mathrm{O}$ & 0.0018 \\
Densidad inicial $\left[\mathrm{Kg} / \mathrm{m}^{3}\right]$ & 1046 & & \\
Temperatura de entrada $[\mathrm{K}]$ & 1026.76 & Temperatura de entrada $[\mathrm{K}]$ & 1239.8 \\
\hline
\end{tabular}


Nótese que a la entrada al horno $(e n z=0$ ) la composición de la fase gaseosa es conocida mientras la de la fase sólida es desconocida (el clinker se obtiene como producto al final del reactor en $\mathrm{z}=\mathrm{L}$ ). Por lo tanto, para dar solución al sistema de ecuaciones diferenciales definido por el modelo unidimensional propuesto y que implica un problema de valor inicial, se debe suponer la composición de entrada de los sólidos. Una vez integradas las ecuaciones hasta la longitud total del reactor, se comparan los valores de composición obtenidos para la fase sólida con los datos de planta (Tabla 4). Si la diferencia entre ellos es menor a una tolerancia establecida (en este caso $1 \times 10^{-3}$ ) se aceptan los resultados, si no se supone de nuevo la composición de los sólidos a la entrada del reactor. Por lo anterior, se utilizó el método matemático del disparo junto con un algoritmo de Newton Raphson para re-estimar los valores supuestos (Chapra y Canale, 2010). Estas dos herramientas fueron implementadas en un código de programación propio utilizando el software MatLab ${ }^{\circledR}$.

\section{RESULTADOS Y DISCUSIÓN}

\section{Perfiles de Composición en el Lecho de Sólidos}

En la Fig. 3 se presenta los perfiles de composición másica desarrollados por los reactivos y productos en el lecho de sólidos y los datos de planta. En esta figura se observa como la composición del CaO permanece prácticamente constante en los primeros 20 metros del reactor rotatorio. Después de este punto, empieza a disminuir debido a que da inicio la formación de Belita $\left(\mathrm{C}_{2} \mathrm{~S}\right.$, reacción 2, Tabla 1). Esto ocurre en mayor proporción que la formación de $\mathrm{CaO}$ por la reacción 1. El avance de la reacción 2 repercute en el decremento en el perfil de composición másica del $\mathrm{SiO}_{2}$, el cual se agota a los 30 metros de longitud de reactor, comportamiento que influye en el perfil desarrollado por el $\mathrm{C}_{2} \mathrm{~S}$ en los primeros 30 metros de longitud del reactor rotatorio. Después de los 30 metros del reactor, la formación de Alita $\left(\mathrm{C}_{3} \mathrm{~S}\right.$, reacción 3 , Tabla 1$)$ ocurre en mayor proporción, generando una disminución en la concentración de $\mathrm{C}_{2} \mathrm{~S}$; por lo tanto, una disminución del perfil de composición de $\mathrm{C}_{2} \mathrm{~S}$ va acompañada con un incremento en el perfil de $\mathrm{C}_{3} \mathrm{~S}$. Las reacciones 3-5, incluidas en la Tabla 1, tienen un avance significativo en los últimos 20 metros de longitud del reactor. $A$ medida que el $\mathrm{CaO}$ se agota, el $\mathrm{Al}_{2} \mathrm{O}_{3}$ y el $\mathrm{Fe}_{2} \mathrm{O}_{3}$ se agotan rápidamente hasta prácticamente desaparecer, lo cual da origen a la formación de los compuestos $\mathrm{C}_{3} \mathrm{~A}$ y $\mathrm{C}_{4} \mathrm{AF}$.
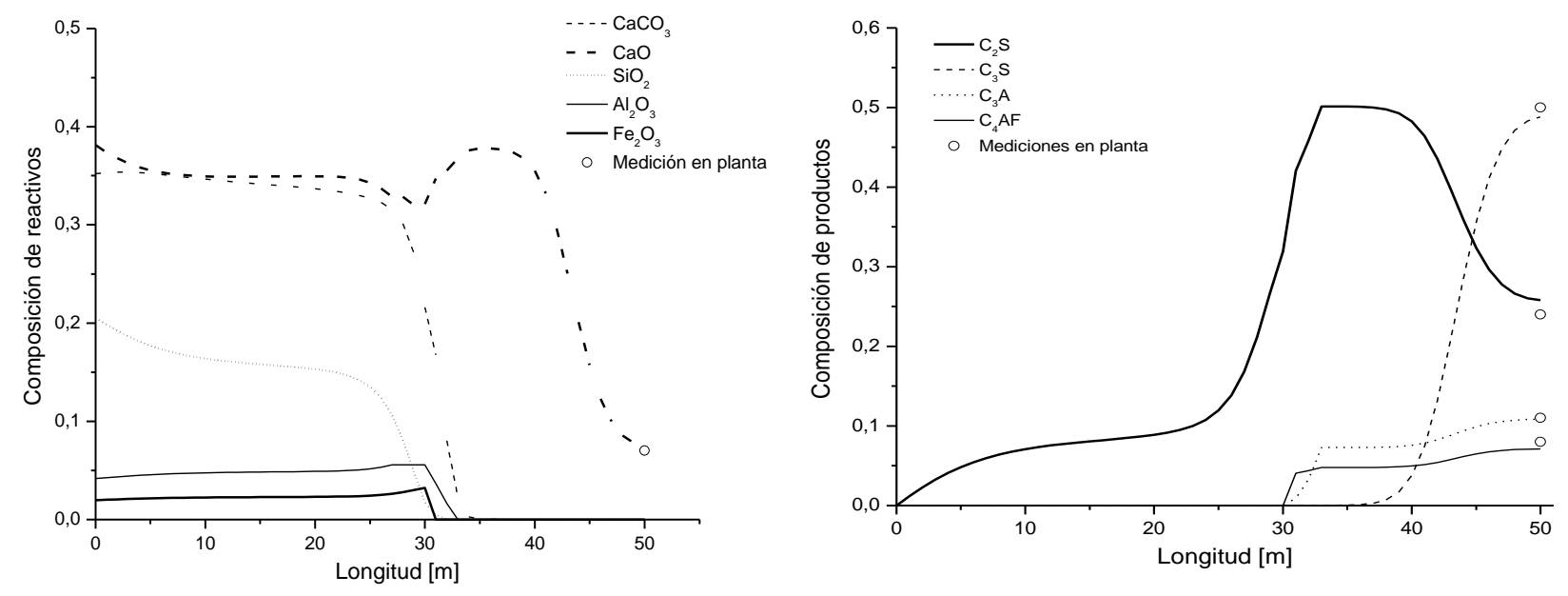

Fig. 3. Perfiles de composición másica de reactivos (izquierda) y de productos (derecha) a lo largo del reactor rotatorio

En la Fig. 4 se presentan los perfiles de composición másica de los gases a lo largo del reactor. Se observa como la composición de $\mathrm{O}_{2}$ y $\mathrm{N}_{2}$ disminuyen debido a la producción de $\mathrm{CO}_{2}$. Comportamiento que se presenta en los primeros 30 metros de longitud ya que la reacción de calcinación de $\mathrm{CaCO}_{3}$ (ver Tabla 1 y Figura 3 izquierda) tiene un notable avance en esa sección del reactor. Por otra parte, la cantidad de vapor de agua producido es bajo, ya que la composición de metano es igualmente baja (consecuencia de la composición del carbón usado como combustible y de la gran cantidad de aire usado en el proceso). Los resultados predichos con el modelo ajustan cuantitativamente bien los datos de planta con un error máximo de $7 \%$. 


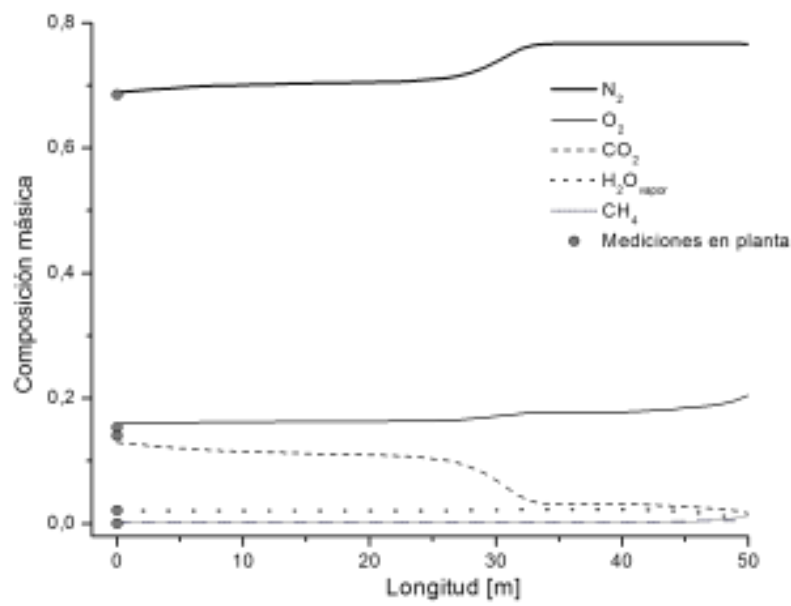

Fig. 4. Perfiles de composiciones másicas de los gases a lo largo del reactor rotatorio

El flujo de sólidos disminuye conforme se avanza en el reactor debido principalmente a la migración de $\mathrm{CO}_{2}$ a la fase gas (Figura 5 derecha). Sin embargo, es posible imaginar la incidencia de otros factores tales como el arrastre de partículas sólidas por el gas, estancamiento del material sólido dentro del equipo, etc... Aun así, el modelo matemático propuesto representa bastante bien la variación del flujo del material sólido a lo largo del reactor rotatorio, con una diferencia entre el valor medido en planta y el valor predicho de aprox. $1.00 \%$. El flujo de $\mathrm{CO}_{2}$ aumenta desde la posición de los quemadores ( $\mathrm{z}=50 \mathrm{~m}$ ) debido a dos factores: la combustión de carbón y las reacciones de calcinación en la fase sólida. Ambas se completan en $2 / 3$ de la longitud total del horno (Figura 5 izquierda).
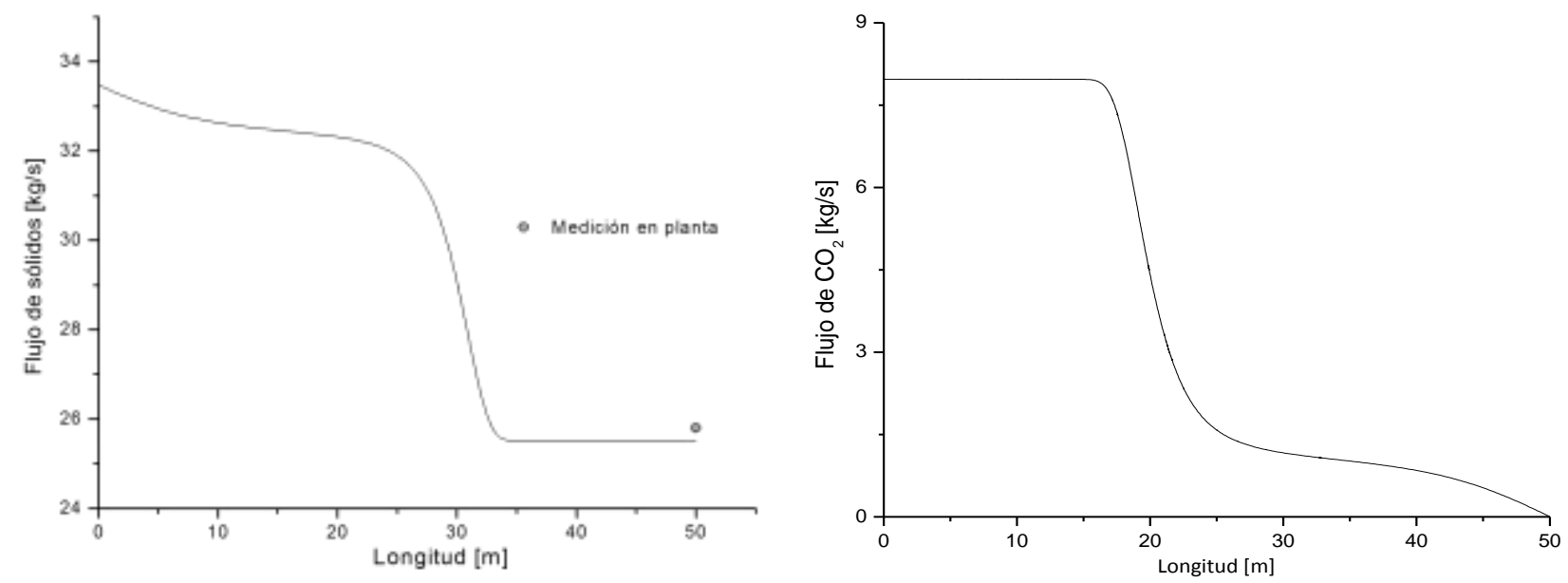

Fig. 5. Perfiles de flujo de sólidos (izquierda) y de $\mathrm{CO}_{2}$ (derecha) a lo largo del reactor rotatorio
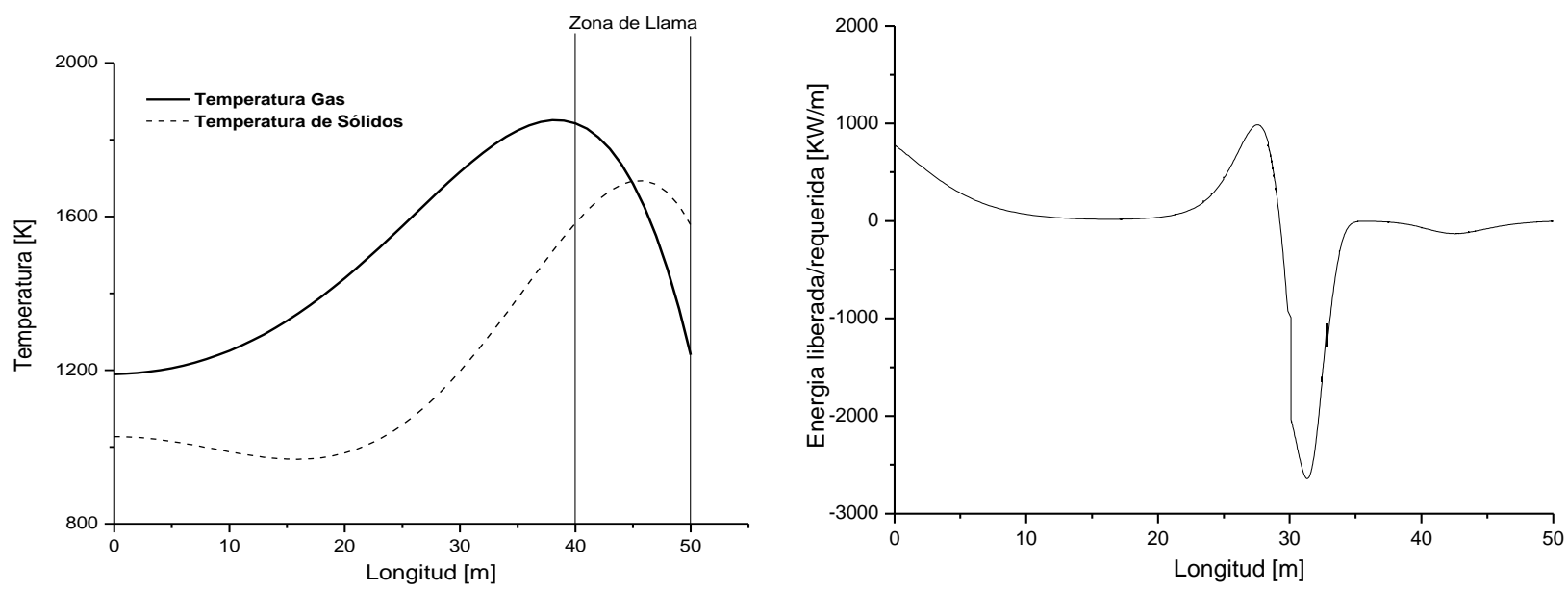

Fig. 6. Izquierda: Perfil de temperatura de sólidos y gases. Derecha: Requerimientos energéticos de las reacciones de clinkerización 
El perfil de temperatura desarrollado por los gases y el carácter dual de las reacciones de clinkerización se evidencia en la Fig. 6 (derecha). Inicialmente (15 metros de longitud), la temperatura del lecho de sólidos disminuye como consecuencia de la baja velocidad de transferencia de energía desde los gases calientes y al elevado requerimiento energético de las reacciones de clinkerización. Entre los 15 y 30 metros de longitud, la transferencia de energía entre los gases calientes y el lecho de sólidos, sumado a la energía liberada por la reacción entre el $\mathrm{CaO}$ y el $\mathrm{SiO}_{2}$, originan un incremento en la temperatura del material sólido. A medida que la temperatura del material sólido se incrementa, las reacciones entre el $\mathrm{CaO}, \mathrm{Al}_{2} \mathrm{O}_{3}$ y $\mathrm{Fe}_{2} \mathrm{O}_{3}$ dan origen a los compuestos $\mathrm{C}_{3} \mathrm{~A}$ y $\mathrm{C}_{4} \mathrm{AF}$. El incremento en la temperatura de los gases en la zona del quemador, se debe a que la velocidad de generación de energía es mucho mayor que la velocidad de transferencia de energía desde los gases a las paredes internas del reactor y al material sólido. En los últimos 10 metros se encuentra el quemador y se define la zona de llama, lugar en el cual se presentan los máximos de temperatura para las dos fases. Perfiles similares han sido reportados por otros autores (Kunii y Chisaki, 2008). Por otra parte, en la Fig. 6 (izquierda) se evidencia cómo varía el carácter térmico de proceso en la fase sólida. Inicialmente endotérmico, presentando un máximo en la demanda de energía causado principalmente por la descomposición de $\mathrm{CaCO}_{3}$ (reacción 1, Tabla 1), luego exotérmico dando una dualidad particular a las reacciones de clinkerización (Cement kilns, 2013). En los últimos 10 metros de longitud del reactor rotatorio, habiendo pasado los picos de temperatura, los requerimientos de energía del sistema reactivo en fase sólida se estabilizan, tendiendo a ser levemente exotérmicas.

\section{CONCLUSIONES}

Se desarrolló un modelo unidimensional en estado estable para el modelamiento y simulación de un reactor rotatorio industrial. Los resultados obtenidos se confrontaron y validaron con mediciones en planta facilitadas por una empresa cementera colombiana, encontrándose un porcentaje de error máximo del $7 \%$. El modelo propuesto, que incluye las cinco principales reacciones de clinkerización, un modelo de reacción gas-sólido para el carbón utilizado como combustible, la reducción de flujo de sólidos a lo largo del equipo debido a la formación de $\mathrm{CO}_{2}$ y la variación de la temperatura del lecho de sólidos y de los gases, permite describir el funcionamiento del reactor. Así se logró cuantificar la variación de los requerimientos energéticos de las reacciones de clinkerización.

\section{NOTACIÓN}

\begin{tabular}{|c|c|}
\hline As: Área ocupada por los sólidos & TG: Temperatura en la fase gas \\
\hline AG: Área ocupada por los gases & $T_{0}$ : Temperatura ambiente \\
\hline $\begin{array}{l}\mathrm{C}_{\mathrm{k}} \text { : Concentración másica del componente } \mathrm{k} \text { en la fase } \\
\text { sólida }\end{array}$ & $\mathrm{T}_{\mathrm{s}}$ : Temperatura en la fase sólida \\
\hline CpiG: Capacidad calorífica de la especie i en la fase gas & $\mathrm{v}_{\mathrm{s}}$ : Velocidad lineal de los sólidos \\
\hline $\mathrm{C}_{\mathrm{ps}}$ : Capacidad calorífica media del lecho de sólidos & $\begin{array}{l}\text { Yis: Composición másica del componente i en la fase } \\
\text { sólida }\end{array}$ \\
\hline D: Diámetro del reactor & YiG: Composición másica del componente i en la fase gas \\
\hline D: Difusividad & UG: Velocidad de flujo de los gases \\
\hline $\mathrm{F}_{\mathrm{CO}_{2}}$ : Flujo másico de $\mathrm{CO}_{2}$ & us: Velocidad de flujo de los sólidos \\
\hline FiG: Flujo másico del componente i en la fase gas & $\begin{array}{l}Z_{\mathrm{ij}:} \text { Coeficiente estequiométrico del componente i en la } \\
\text { reacción j en la fase sólida }\end{array}$ \\
\hline$F_{s}:$ Flujo másico de material sólido & $\Delta \mathrm{H}_{\mathrm{rxn}}$ : Entalpía de la reacción i en la fase sólida \\
\hline $\mathrm{h}_{\mathrm{s}}$ : Altura del material sólido & $\Delta \mathrm{H}_{\mathrm{rxn}_{\mathrm{iG}}}$ : Entalpía de reacción en la fase gas \\
\hline \multicolumn{2}{|l|}{$\mathrm{H}_{\mathrm{CO}_{2}}$ : Entalpía de $\mathrm{CO}_{2}$} \\
\hline kG: Conductividad térmica del gas & Símbolos griegos \\
\hline \multicolumn{2}{|l|}{$\begin{array}{l}\text { Ls: Longitud de la cuerda de sólidos expuesta a los } \\
\text { gases calientes }\end{array}$} \\
\hline $\mathrm{M}_{\mathrm{i}}$ : Peso molecular del compuesto i & $\alpha_{G}$ : Absortividad del gas \\
\hline Un: Número de Nusselt & $\beta$ : Ángulo de inclinación del reactor \\
\hline Po2: Presión parcial de oxígeno & $\omega$ : Velocidad de rotación del reactor \\
\hline Q: Flujo de calor entre los gases y el lecho de sólidos & @: Ángulo de reposo del material sólido \\
\hline qs: Caudal volumétrico de sólidos & $\rho_{s}:$ Densidad del sólido \\
\hline R: Radio interno del reactor & $\rho G:$ Densidad del gas \\
\hline r: Radio de giro de las partículas sólidas & $\lambda_{\mathrm{ml}}$ : Calor latente de fusión \\
\hline ro: radio mínimo de giro de las partículas sólidas & $\mu \mathrm{G}:$ Viscosidad del gas \\
\hline $\begin{array}{l}\text { Ris: Velocidad de reacción del componente i en la fase } \\
\text { sólida }\end{array}$ & $\sigma:$ Constante de Stefan-Boltzmann \\
\hline $\begin{array}{l}\mathrm{R}_{\mathrm{iG}} \text { : Velocidad de reacción del componente i en la fase } \\
\text { gas }\end{array}$ & $\varepsilon$ : Emisividad \\
\hline
\end{tabular}




\section{AGRADECIMIENTOS}

Se agradece a Javier Fontalvo por sus sugerencias académicas. Así mismo, a COLCIENCIAS y la Universidad Nacional de Colombia, sede Manizales por el aporte económico para desarrollar esta investigación (Programa Jóvenes Investigadores e Innovadores 2009 Convenio interadministrativo especial de cooperación 704).

\section{REFERENCIAS}

astm.org, http://www.astm.org/Standards/C150C150M-SP.htm. Acceso: 27 de noviembre (2013).

Cement kilns, http://www.cementkilns.co.uk/ckr_therm.html. Acceso: 26 de noviembre (2013).

Coz Díaz, J.J., y otros tres autores, Design and finite element analysis of a wet cycle cement rotary kiln, Finite Elements in Analysis and Design: 39, 17-42 (2002).

Chapra, S. C. y R. P. Canale, Numerical methods for engineers, 6a edición, 780-783. McGraw Hill, New York, Estados Unidos (2010).

European Commission. Reference document on best available techniques in cement, lime and magnesium oxide manufacturing industries, http://www.umweltbundesamt.de/sites/default/files/medien/419/ dokumente/ clm_bref_0510.pdf. Acceso: 20 de noviembre (2013).

Fidaros, D.K., y otros tres autores, Numerical modelling of flow and transport processes in a calciner for cement production, Powder Technology: 171, 81-95 (2007).

Froment, G. F., K. B. Bischoff y J. De Wilde, Chemical Reactor Analysis and Design,3a edición, 505-513. Wiley, Hoboken, New Jersey, Estados Unidos (2011).

Hewlett, P. C., Lea's Chemistry of Cement and Concrete, 4a edición, 95-126. Elsevier Butterworth-Heinemann, Oxford, Inglaterra (1988).

Ishii, K., Advance pulverized coal injection technology and blast furnace operation, $1^{\text {a }}$ edición, 37-62. Elsevier, Amsterdam, Holanda (2000).

Kunii, D. y T. Chisaki, Rotary Reactor Engineering, 11-68. Elsevier, Amsterdam, Holanda (2008).

Levenspiel, O. Chemical Reaction Engineering, 3a edición, 570-582. Wiley, Hoboken, New Jersey, Estados Unidos (1999).

Li, S.-Q. y otros tres autores, A Mathematical Model of Heat Transfer in a Rotary Kiln Thermo-Reactor. Chemical Engineering \& Technology: 28, 1480 -1489 (2005).

Martins, M.A., L.S. Olivera y A.F. Franca, Modeling and simulation of petroleum coke calcination in rotary kilns, Fuel: 80, 1611-1622 (2001).

Mastorakos, E. y otros cinco autores, CFD predictions for cement kilns including flame modelling, heat transfer and clinker chemistry, Applied Mathematical Modelling: 23, 55-76 (1999).

Mujumdar, K.S., A. Arora y V.V. Ranade, Modeling of Rotary Cement Kilns: Applications to Reduction in Energy Consumption, Industrial and Engineering Chemistry Research: 45, 2315-2330 (2006).

Mujumdar, K.S. y V.V. Ranade, CFD modeling of rotary cement kilns, Asia-Pacific Journal of Chemical Engineering: 3, 106-118 (2008).

Schaffel, N., y otros tres autores, Mathematical modeling of MILD combustion of pulverized coal, Combustion and Flame: 156, 1771-1784 (2009).

Taylor, H.F.W., Cement Chemistry, 2ª edición, 1-33. Thomas Telford Publishing, Londres, Inglaterra (1997). 